\title{
A Similarity Subgrid Model for Premixed Turbulent Combustion
}

\author{
A. W. Vreman • R. J. M. Bastiaans • B. J. Geurts
}

Received: 10 January 2008 / Accepted: 4 August 2008 / Published online: 9 September 2008

(C) The Author(s) 2008. This article is published with open access at Springerlink.com

\begin{abstract}
The accuracy of large-eddy simulation (LES) of a turbulent premixed Bunsen flame is investigated in this paper. To distinguish between discretization and modeling errors, multiple LES, using different grid sizes $h$ but the same filterwidth $\Delta$, are compared with the direct numerical simulation (DNS). In addition, LES using various values of $\Delta$ but the same ratio $\Delta / h$ are compared. The chemistry in the LES and DNS is parametrized with the standard steady premixed flamelet for stochiometric methane-air combustion. The subgrid terms are closed with an eddyviscosity or eddy-diffusivity approach, with an exception of the dominant subgrid term, which is the subgrid part of the chemical source term. The latter subgrid contribution is modeled by a similarity model based upon $2 \Delta$, which is found to be superior to such a model based upon $\Delta$. Using the $2 \Delta$ similarity model for the subgrid chemistry the LES produces good results, certainly in view of the fact that the LES is completely wrong if the subgrid chemistry model is omitted. The grid refinements of the LES show that the results for $\Delta=h$ do depend on the numerical scheme, much more than for $h=\Delta / 2$ and $h=\Delta / 4$. Nevertheless, modeling errors
\end{abstract}

\footnotetext{
A. W. Vreman · R. J. M. Bastiaans $(\varangle)$

Combustion Technology, Department of Mechanical Engineering,

Eindhoven University of Technology, P.O. Box 513,

5600 MB Eindhoven, The Netherlands

e-mail: R.J.M.Bastiaans@tue.nl
}

\author{
A. W. Vreman \\ Vreman Research, Godfried Bomansstraat 46, \\ 7552 NT Hengelo, The Netherlands
}

\section{B. J. Geurts}

Mathematical Sciences, University of Twente,

P.O. Box 217, 7500 AE Enschede, The Netherlands

B. J. Geurts

Applied Physics, Eindhoven University of Technology,

P.O. Box 513, 5600 MB Eindhoven, The Netherlands 
and discretization error may partially cancel each other; occasionally the $\Delta=h$ results were more accurate than the $h \leq \Delta$ results. Finally, for this flame LES results obtained with the present similarity model are shown to be slightly better than those obtained with standard $\beta$-pdf closure for the subgrid chemistry.

Keywords Large-eddy simulation • Similarity models • Turbulent combustion • Premixed flamelets

\section{Introduction}

Large-Eddy Simulation (LES) of combustion as research topic has gained an increasing amount of attention in recent years. The subject is complicated, because questions regarding LES methodology and modeling issues related to chemistry need to be considered simultaneously. In order to be able to perform three-dimensional time-dependent simulations of turbulent flows with combustion, it is usually not realistic to solve transport equations for all species occurring in the chemical reaction process. Therefore, it is common to apply a reduction technique to limit the number of transport equations that need to be carried in three dimensions. One group of reduction techniques is formed by the flamelet approaches [1-6]. In flamelet approaches, results from one-dimensional computations with detailed chemistry (flamelets) are mapped to one or a few representative variables. Subsequently, the mapping functions, which are represented by a one- or multidimensional table, are used in the three-dimensional computation of a specific application.

LES of turbulent combustion is complicated, because in most cases neither turbulent structures nor the thickness of the flame can be properly resolved on the computational mesh. Thus, in addition to subgrid modeling of the turbulence, subgrid modeling of the chemical reaction process, which appears in the equations as one or more nonlinear source-terms, needs to be considered. The purpose of the present paper is to develop LES for turbulent combustion further and to study its accuracy. For the latter purpose we follow the strategy proposed in Ref. [7] (and revisited for example in Ref. [8]) and perform LES for multiple mesh sizes $h \leq \Delta$, where the filterwidth $\Delta$ is held fixed. Modeling and discretization errors are thus separated, since discretization errors reduce if $h$ is reduced and $\Delta$, the length-scale of the smallest resolved structures in the simulation, is kept constant.

To test LES of turbulent combustion we have chosen a premixed Bunsen flame, similar to experiments by Filatyev et al. [9] and simulations by Bell et al. [10]. The size of the flame is somewhat smaller in our case, to enable well-resolved Direct Numerical Simulation (DNS) with moderate computational effort. In the present paper both DNS and LES assume flamelet chemistry, but unlike LES, the DNS resolves both flame thickness and turbulence down to the Kolmogorov length-scale. Therefore the DNS does not require subgrid modeling, and it can be used to test and develop LES models.

The flamelet chemistry will be based on an unstretched flamelet. This is a reasonable assumption if the flame is in the thin reaction zone, see Ref. [11], where simulations based on an unstretched flamelet have been shown to capture stretched flames reasonably accurately, provided they are in that zone. We will verify that 
the turbulent intensity in the present case is low enough to let the flame be in the thin reaction zone. A Bunsen flame with a higher turbulent intensity might access the broken reaction zone, where stretch may quench the flame locally, and then a simulation based on non-stretched flamelets is less likely to be applicable. However, many industrial applications are in the thin reaction zone and thus it is legitimate to develop and validate LES with use of a DNS that is performed with a chemical reduction technique that may be limited to the thin reaction zone.

The type of LES model for the subgrid reaction effects will be based on similarity principles (the model will be compared with standard $\beta$-pdf modeling). To address the applicability of a similarity model for subgrid chemistry, we need to compare the important physical length-scales of the flame with the LES filterwidth $\Delta$. The main value that we choose for the LES filterwidth $\Delta(0.8 \mathrm{~mm})$ is twice as large than the thickness of the preheat zone. The latter is close to the flame thickness based on maximum gradient of either temperature or progress variable (both slightly smaller than $0.4 \mathrm{~mm}$ ). The thickness of the thin reaction zone is 3 to 10 times thinner than the preheat zone. Thus the reaction zone is an order of magnitude smaller than the filterwidth. LES is often thought to face the same problems as RANS in these cases $[12,13]$, but such statements have not been quantified by numbers sofar. In this paper we will investigate to which extent it is possible to perform LES for a case with length-scales and filterwidth just mentioned. In such a case the use of a similarity hypothesis is controversial [12], since the reaction zone may be entirely subgrid, thus to draw information from resolved scales about the reaction zone seems impossible. Therefore, we give two arguments why the similarity approach adopted in the present paper is justified none the less.

First, the present LES is combined with a premixed flamelet approach. In this flamelet approach all chemistry is mapped upon the progress variable (and later on only post-processing is needed to retrieve the concentrations). Since the progress variable is the only scalar carried in the $3 \mathrm{D}$ simulation, it is the resolution of the progress variable that counts to judge the applicability of models. The progress variable is chosen such that it spans the entire reaction process and consequently its variation is relatively slow and the thickness of its jump across the flame is relatively large. In this sense the resolution requirements for the flamelet approach are less strict than for simulations that carry the detailed chemistry in the 3D transport equations, since in the latter case the resolution requirements have to be based on the species with the thinnest structure.

Second, the filterwidth adopted is only twice as large as the thickness of the progress variable, which is nevertheless sufficient for the LES to be three orders of magnitude cheaper than the DNS. For suitable filters, this does not mean that the reaction is entirely subgrid (although most of it will be). In contrast to a sharp cut-off filter, the resolved scales of top-hat or Gaussian filtered fields still contain significant information of scales that are a few times smaller. We will demonstrate that it is possible to use similarity or deconvolution modeling based on top-hat filtering to represent the subgrid information required. However, we will see that the width of the similarity filter should be larger than the basic filter width, to extract sufficient information from the smallest resolved scales.

The outline of the paper is as follows. In Section 2 we specify the governing equations, the approach of subgrid modeling, and the numerical discretization. In Section 3 results are shown for the premixed Bunsen flame and LES with the 
similarity model is compared to DNS. Also a comparison with the standard $\beta$-pdf model is included. Conclusions are summarized in Section 4.

\section{Computational Method}

\subsection{The unfiltered equations}

The Navier-Stokes equations with parametrized premixed chemistry read:

$$
\begin{aligned}
\frac{\partial \rho}{\partial t}+\frac{\partial \rho u_{j}}{\partial x_{j}} & =0 \\
\frac{\partial \rho u_{i}}{\partial t}+\frac{\partial \rho u_{i} u_{j}}{\partial x_{j}} & =-\frac{\partial p}{\partial x_{i}}+2 \frac{\partial \mu S_{i j}}{\partial x_{j}} \\
\frac{\partial \rho c}{\partial t}+\frac{\partial \rho u_{j} c}{\partial x_{j}} & =\frac{\partial}{\partial x_{j}}\left(\rho D \frac{\partial c}{\partial x_{j}}\right)+\omega_{c}, \\
\rho & =f_{1}(c), \\
T & =f_{2}(c), \\
\omega_{c} & =f_{3}(c),
\end{aligned}
$$

where the summation convention over repeated indices is used, while $\rho, \mathbf{u}, p, T$, and $c$ represent density, velocity vector, pressure, temperature, and progress variable. The progress variable is based upon the scaled mass fraction of $\mathrm{O}_{2}$ and is scaled such that it equals zero in the unburnt and one in the burnt regions. This mass fraction is selected as progress variable because, unlike many other species $\left(\mathrm{CO}_{2}\right.$ for example), the mass fraction of $\mathrm{O}_{2}$ satisfies the requirement of strict monotonicity on the entire flamelet. The functions $f_{j}$ (plotted in Fig. 1) denote the quantities that are retrieved from the flamelet database. The flamelet database is composed of a single flamelet, obtained by solving the premixed flamelet equations with detailed chemistry using the GRI 3.0 reaction scheme for a stoichiometric methane-air mixture [4]. The specific choice of the progress variable (here $\mathrm{O}_{2}$ ) determines the precise shapes of the functions $f_{j}$. The relatively large increase of $T$ for $c>0.9$ is probably caused by the larger importance of the oxidation with $\mathrm{O}$ in the later stages of the reaction (when there is not much $\mathrm{O}_{2}$ left).

It is remarked that $T$ is almost reversely proportional to $\rho ; T$ is prescribed by the combustion approximation, an approximate equation of state [4],

$$
\rho=\frac{p_{a m b}}{R T \sum_{i=1}^{N_{s}} Y_{i} / M_{i}},
$$

where $p_{a m b}$ is the ambient pressure (which is constant), $R$ the universal gas constant and $N_{s}=53$ the total number of species in the detailed chemistry reaction scheme, while $Y_{i}$ and $M_{i}$ are the mass fraction and molar mass of species $i$ in the flamelet, respectively.

In addition the rate of strain is defined by

$$
S_{i j}=\frac{1}{2}\left(\frac{\partial u_{i}}{\partial x_{j}}+\frac{\partial u_{j}}{\partial x_{i}}-\frac{2}{3} \frac{\partial u_{k}}{\partial x_{k}} \delta_{i j}\right),
$$



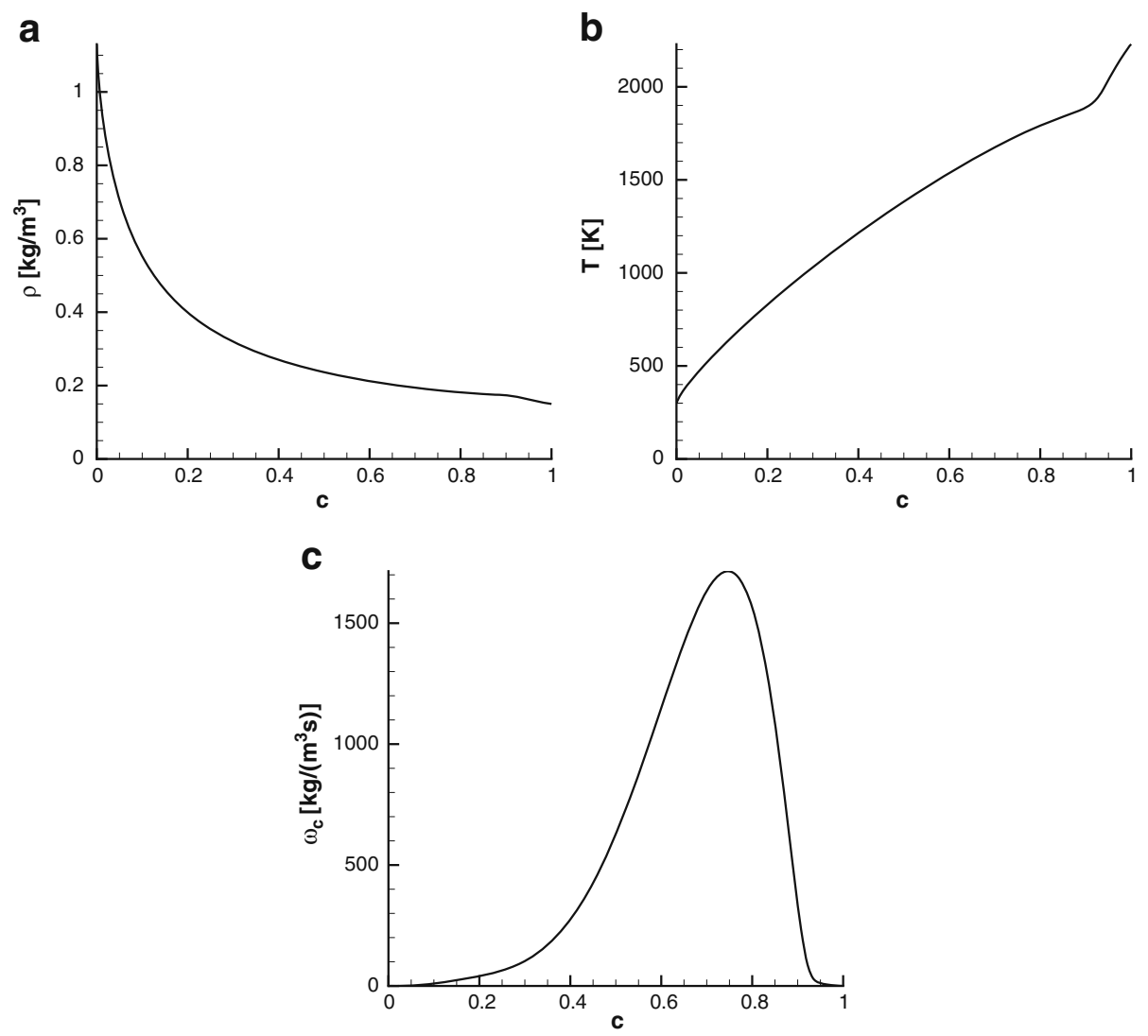

Fig. 1 Visualization of the flamelet database (functions $f_{i}(c)$ ): density (a), temperature $(\mathbf{b})$ and chemical source term $(\mathbf{c})$

while the viscosity $\mu$ is a function of temperature according to Sutherland's threecoefficient law. The thermal diff usivity is equal to

$$
\lambda / c_{p}=2.58 \cdot 10^{-5}(T / 298 \mathrm{~K})^{0.69},
$$

where $\lambda$ is the thermal conductivity and $c_{p}$ the specific heat [14]. The diff usivity $\rho D_{c}$ is equal to $\lambda / c_{p}$ divided by the Lewis number of $\mathrm{O}_{2}$ (1.11).

\subsection{Subgrid modeling}

Whereas DNS with flamelet chemistry solves the unfiltered equations, LES solves the filtered equations. The basic filter definition in LES is given by $\bar{\rho}=G \rho$, where $G$ is the filter operator, a convolution integral operator with top-hat filter kernel with width $\Delta$. In variable density flows it is convenient to use the density-weighted or Favre filter as well, defined by $\tilde{u}_{i}=\overline{\rho u}_{i} / \bar{\rho}$.

The filtered equations are obtained by application of the basic filter operations to the equations in the previous subsection. The nonlinearities in the equations lead to unknown terms, which are either modeled, or neglected. Subgrid terms arising 
from the nonlinearity of the viscous terms are neglected, while the subgrid terms arising from the convective terms are closed by adding an eddy-viscosity $\mu_{t}$ to the molecular viscosity in the momentum equations and an eddy-diffusivity $\mu_{t} / S c_{t}$ with $S c_{t}=0.4$ [2] to the molecular diffusivity in the scalar equation. For the eddy-viscosity the following model is used [15]:

$$
\mu_{t}=\bar{\rho} C\left(\frac{\beta_{11} \beta_{22}-\beta_{12}^{2}+\beta_{11} \beta_{33}-\beta_{13}^{2}+\beta_{22} \beta_{33}-\beta_{23}^{2}}{\alpha_{k l} \alpha_{k l}}\right)^{1 / 2} .
$$

Here the tensor $\beta$ equals the gradient model:

$$
\beta_{i j}=\Delta_{k} \alpha_{k i} \alpha_{k j}, \quad \alpha_{k i}=\frac{\partial \tilde{u}_{i}}{\partial x_{k}}
$$

where $\Delta_{k}$ is the filterwidth in direction $x_{k} ; \Delta_{1}=\Delta_{2}=\Delta_{3}=\Delta$ is used throughout this paper. The model constant is related to the Smagorinsky constant $C=2.5 C_{S}^{2}$; in this work we take $C=0.025$. Model (10) appeared to be as accurate as the dynamic subgrid model in non-reacting wall-bounded and free shear flow [15].

Due to the nonlinearity of the chemical parametrization (nonlinearities in $f_{1}$ to $f_{3}$ ), non-standard subgrid terms arise, $\overline{f_{i}(c)}-f_{i}(\tilde{c})$. Geurts [17] proposed to model these non-standard terms with approximate deconvolution. Here we use a similar strategy, but in order to compensate for the fact that a deconvolution retrieves resolved scales only, we slightly alter the common methodology and apply the deconvolution at a larger scale; the filterwidth of the 'inverted' filter now equals $2 \Delta$. We define a deconvoluted scalar by

$$
c \approx 2 \tilde{c}-J \tilde{c}
$$

where

$$
J \tilde{c}=\frac{H(\bar{\rho} \tilde{c})}{H(\bar{\rho})} .
$$

is the Favre filter associated with the top-hat filter $H, H$ being defined with filterwidth $2 \Delta$. Since by definition $\rho=f_{1}(c)$, Eq. 12 is an implicit equation for $c$. To simplify this problem, we use $\rho$ at the previous time-level $\left(f_{1}\left(c_{\text {old }}\right)\right)$ to evaluate $J$, thereby introducing an error of $O(\delta t)$ where $\delta t$ is the time-step.

In fact $2 \tilde{c}-J \tilde{c}$ in Eq. 12 can be expressed as $c=\tilde{c}+c^{\prime \prime}$ in case the subgrid fluctuation $c^{\prime \prime}$ is modeled by $\tilde{c}-J \tilde{c}$. This is a scale-similarity hypothesis [18], and thus Eq. 16 is essentially a similarity model. However, $\tilde{c}-J \tilde{c}$ is also the first term in the series expansion of the deconvolution operator [19]. It is remarked that the deconvolution approach is applicable to a Favre filter, since a Favre filter can be written as an integral operator with a positive filter kernel [20]. To extend the deconvolution, more terms may be added to approximate $c$, but we do not pursue this here.

Using the similarity deconvolution, the unknown quantities $\overline{f_{i}(c)}$ are modeled by

$$
\begin{aligned}
\bar{\rho} & =H f_{1}(2 \tilde{c}-J \tilde{c}), \\
\tilde{T} & =\frac{H\left(\rho f_{2}(2 \tilde{c}-J \tilde{c})\right)}{H \rho}, \\
\overline{\omega_{c}} & =b(\tilde{c}) H f_{3}(2 \tilde{c}-J \tilde{c}),
\end{aligned}
$$


Here $b(\tilde{c})$ is a blending function which is approximately 1 in the main part of the domain, but zero at the end-points of the interval $[0,1]$. This blending function is needed to ensure realizability $(0 \leq c \leq 1)$ that may be violated by numerical discretization of the filters in the model above. The numerical discretization of the filter introduces a stencil with a certain width to evaluate for example $\bar{\omega}_{c}$. Because this stencil involves neighboring points, the source term $\mathrm{Hf}_{3}$ is not zero in all grid points where $\tilde{c}$ equals 1 . In grid points where $\tilde{c}$ equals 1 , while $H f_{3}$ is nonzero, $\tilde{c}$ will become larger than 1 at the next time-step, thus violating the physical upperbound of $\tilde{c}$. The blending function is defined by

$$
b(\tilde{c})=\left(1-\tilde{c}^{n}\right)\left(1-(1-\tilde{c})^{n}\right),
$$

if $0<\tilde{c}<1$ and otherwise $b=0$. The blending function is continuous while for $n>$ 100 , it satisfies $0.99<b \leq 1$ for $0.05<\tilde{c}<0.95$. Figure 2 shows the global spatial maximum of the progress variable as a function of time for two versions of LES1a (defined later on) LES using the similarity model with and without blending function. Without blending function overshoots of the progress variable occur. With blending function overshoots do not occur, such that no clipping of the progress variable is needed to satisfy $0 \leq \tilde{c} \leq 1$. Except from Fig. 2, all results shown for the similarity model have been obtained with the blending function switched on. For the exponent $n$ in the blending function a value of 100 is used, which is sufficiently high (we verified that statistics plotted for two versions of LES1a $(n=100$ and $n=200)$ were on top of each other).

The similarity model for $\bar{\rho}, \tilde{T}$ and $\overline{\omega_{c}}$ will be compared with the presumed $\beta$-pdf model for these quantities $[2,5,6,21,22]$. For the simulation with the presumed $\beta$ pdf model a manifold with an additional entry for the subgrid variance $\widetilde{c^{2}}-\tilde{c}^{2}$ is used. For each $\tilde{c}$ in the manifold the subgrid variance is discretized between 0 and $\tilde{c}(1-\tilde{c})$ using 100 uniform steps. In the three-dimensional simulation the subgrid variance is

Fig. 2 Spatial maximum of the instantaneous progress variable as a function of time. LES1a with (solid) and without blending function (dashed)

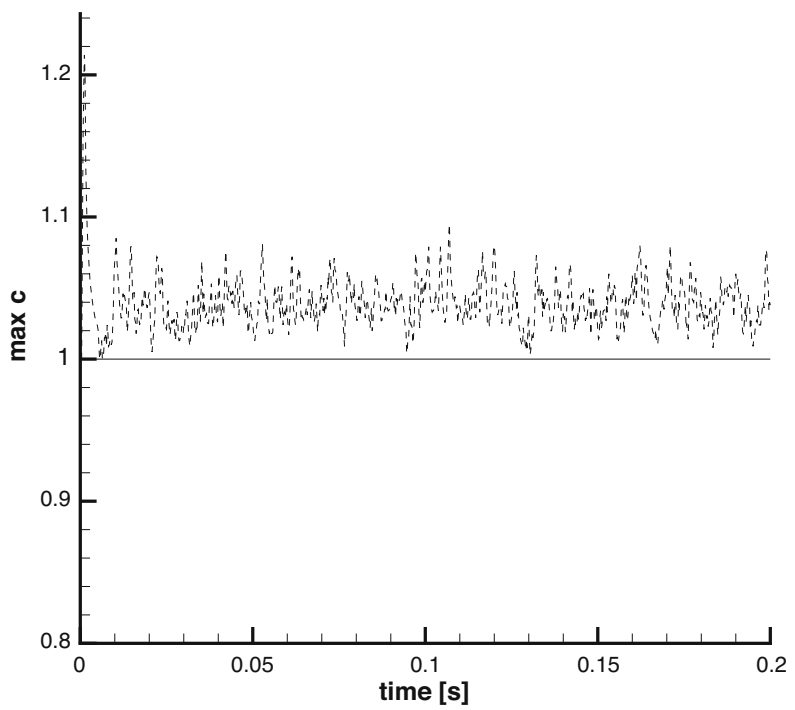


modeled by $a \Delta^{2}|\nabla \tilde{c}|^{2}$, where $a=0.14$, which falls in the range of values produced by a dynamic model for $a$ (typically between 0.1 and 0.2 [23]).

Both the proposed similarity model and the $\beta$-pdf model may enter the manifold with 'unrealizable' values; the deconvolved quantity $2 \tilde{c}-J \tilde{c}$ may attain values larger than 1 or smaller than 0 ; the modeled $\beta$-pdf variance may attain values larger than the theoretical maximum $\beta$-pdf variance, $\tilde{c}(1-\tilde{c})$. The manifold functions are therefore extended beyond their formal domain by constant extrapolation of the values at the boundaries. The fractions of grid points for which this extrapolation was actually used will be given in Section 3.2.

\subsection{Numerics}

We used a straightforward and efficient numerical implementation to solve the equations with parametrized chemistry. The variable density approach involved a Poisson equation for the pressure, similar to other low-Mach methods [2,5].

For the continuity and momentum equations the standard finite volume method was employed, with second-order central differencing on a staggered Cartesian mesh, while for the scalar an upwind method was used. To improve stability an hybrid explicit scheme was selected to integrate the momentum equations: AdamsBashforth for the convective and forward Euler for the viscous terms. The scalar was updated with pure forward Euler since for an upwind method the hybrid timestepping scheme has less advantages than for central differencing. In more detail the algorithm that is used to update the variables from level $n$ to $n+1$ consists of five steps, listed first in Ref. [16] and reproduced below for clarity.

The first step is to obtain $c^{n+1}$. For this purpose the scalar equation was recast into the equivalent advective formulation:

$$
\frac{\partial c}{\partial t}+u_{j} \frac{\partial c}{\partial x_{j}}=\left[\frac{\partial}{\partial x_{j}}\left(\rho D \frac{\partial c}{\partial x_{j}}\right)+\omega_{c}\right] / \rho,
$$

In contrast to discretization of the conservative form of the convective term, discretization of the advective form does not require $\rho^{n+1}$ to find $c^{n+1}$. For the discretization of the advective term in the scalar equation the Van Leer third-order accurate MUSCL scheme [24], which is TVD, is applied. Thus unlike the spatial discretization of the momentum equation, the spatial discretization of the scalar equation introduces numerical diffusion. For the scalar equation numerical diffusion is hard to avoid if we want to keep the scalars in between their physical bounds on coarse grids.

In the second step we compute the viscous minus convective terms in the momentum equation, their sum is denoted by $\mathbf{q}$,

$$
q_{i}=\left(\frac{\partial 2 \mu S_{i j}}{\partial x_{j}}\right)_{n}-\frac{3}{2}\left(\frac{\partial \rho u_{i} u_{j}}{\partial x_{j}}\right)_{n}+\frac{1}{2}\left(\frac{\partial \rho u_{i} u_{j}}{\partial x_{j}}\right)_{n-1},
$$

and we obtain the uncorrected momentum by

$$
\mathbf{w}=\hat{\rho}^{n} \mathbf{u}^{n}+\delta t \mathbf{q}
$$

where $\delta t$ is the time-step, and $\hat{\rho}$, the average of the density of the two nearest neighboring points, needed to obtain the density at the staggered velocity location in between. The convective momentum fluxes on the faces of the staggered cells 
are computed as the products of velocities multiplied with the density on the faces. However, the density is defined at the pressure cell centers (like the scalars). Averages involving the smallest possible number of density cells are used to obtain the density on the faces of the velocity cells. For example, if we compute the momentum flux through the $x_{1}$ face of the $u_{1}$ cell, the density is available right there; no density interpolation is required. But if we compute the momentum flux through $x_{2}$ or $x_{3}$ faces of the $u_{1}$ cell the density needs to be interpolated from four points corresponding to the corners of a surrounding square.

The third step is to calculate $\rho^{n+1}, T^{n+1}$ and $w_{c}^{n+1}$ from the flamelet database with entry $c^{n+1}$ (using linear interpolation of the values in the table).

In the fourth step a Poisson equation is solved to obtain the pressure. To obtain the Poisson equation, the unknown momentum at level $n+1$,

$$
\hat{\rho}^{n+1} \mathbf{u}^{n+1}=\mathbf{w}-\nabla(p \delta t),
$$

is substituted into the continuity equation

$$
\frac{\partial \hat{\rho}^{n+1} u_{i}^{n+1}}{\partial x_{i}}=-\frac{\rho^{n+1}-\rho^{n}}{\delta t} .
$$

Thus the Poisson equation reads

$$
\frac{\partial^{2}(p \delta t)}{\partial x_{i}^{2}}=\frac{\rho^{n+1}-\rho^{n}}{\delta t}+\frac{\partial w_{i}}{\partial x_{i}} .
$$

It is solved by a multigrid method using $V$-cycles and the SOR smoother (lexicographical Gauss-Seidel with overrelaxation factor 1.5). The restriction operator is the uniform average from eight small cells to a large cell, while trilinear interpolation [25] is used as prolongation operator.

The fifth and last step provides the momentum at the new time level by evaluating Eq. 21 from which the velocity at the new time level directly follows.

\section{Results}

\subsection{Flow conditions}

In this section we present DNS and LES results for a planar Bunsen flame. The configuration of the premixed stoichiometric methane-air Bunsen flame simulated was a spatial planar jet of the unburnt mixture (mean centerline velocity $U_{0}=3 \mathrm{~m} / \mathrm{s}$ and $T=298 \mathrm{~K}$ ), surrounded by a co-flow with hot products (velocity $7 \mathrm{~m} / \mathrm{s}$ and $T=2240 \mathrm{~K}$ ). The slot width of the burner equalled $8 \mathrm{~mm}$, different from otherwise similar experiments [9] and simulations [10,16].

The DNS was performed on a uniform grid with 320 points in the inhomogeneous streamwise direction $(z), 256$ points in the inhomogeneous normal direction $(y)$, and 160 points in the spanwise homogeneous (periodic) direction $(x)$. Each grid cell was cubic and the grid size was $0.1 \mathrm{~mm}$. The time step of the DNS, $\delta t=0.1 \cdot 10^{-5} \mathrm{~s}$, was verified to be sufficiently small (Fig. 3a shows contours for this DNS and a DNS with $\delta t$ twice as large. The contours can hardly be distinguished.) The outflow boundary conditions in the normal and streamwise direction assumed Neumann conditions for the three velocity components. The pressure satisfied Neumann conditions at the 
Fig. 3 Isocontours $0.2,0.4,0.6$ and 0.8 of the (mean) progress variable: DNS turbulent Bunsen flame (a) and laminar Bunsen flame (b). The solid lines in a were obtained with $\delta t=1.0 \cdot 10^{-5} \mathrm{~s}$, dashed lines with $\delta t=2.0 \cdot 10^{-5} \mathrm{~s}$ (almost on top of the solid lines)

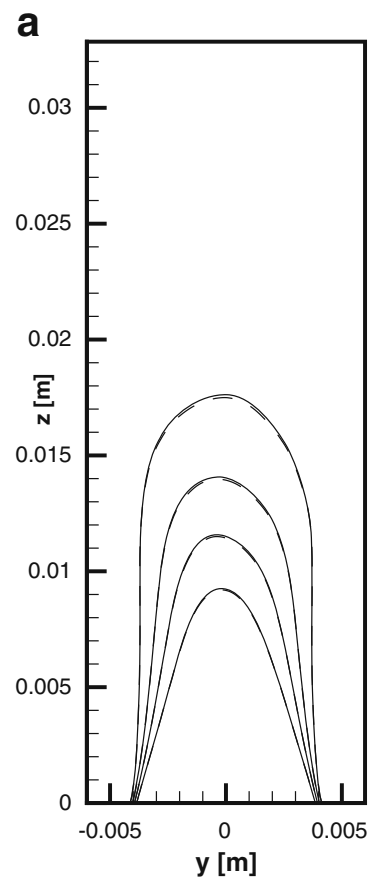

streamwise in- and outflow, while it was held constant in the normal outflow planes. The mean inflow profiles were based on tangent hyperbolic profiles with a thickness of $0.384 \mathrm{~mm}$ (based on maximum derivative).

In the experiments by Filatyev et al. [9] the inflow turbulence was generated by a suitable grid that set the length-scale of the turbulence. For the present simulation results, the velocity at the core of the inflow was perturbed with random uniform noise, which was filtered to control the turbulence length-scale. No inflow perturbations were added to the mean of the progress variable. The inflow velocity perturbations were constructed such that they were the same for each simulation in the present section, both DNS and LES. For each velocity component, and each $\delta t_{0}=10^{-6} \mathrm{~s}$, random numbers between -1 and 1 were generated on the DNS inflow plane $(160 \times 256)$. Then these random numbers were filtered, applying a box-filter of $l_{0}=5.2 \mathrm{~mm}$ in the spatial directions, and a temporal exponential filter. For a signal $q$, the temporal filter was discretized by

$$
\hat{q}_{n+1}=\left(1-\frac{\delta t_{0} \sqrt{12}}{l_{0} / U_{0}}\right) \hat{q}_{n}+\frac{\delta t_{0} \sqrt{12}}{l_{0} / U_{0}} q_{n} .
$$

The perturbation was initialized with $\hat{q}_{0}=0$. The width of this temporal filter based on its second-order moment equals $l_{0} / U_{0}$. Subsequently, periodic boundary conditions were imposed in both spatial directions, and then the spatial filter with length $l_{0}$ was performed. After the filtering, the inflow was multiplied with 1650 (to obtain the inflow intensities mentioned later on) and confined to the center jet (using a tangent hyperbolic function of the same shape as the mean inflow profiles). Finally, 
for the LES, the discrete inflow plane was injected to the simulation grid of each specific case.

A laminar simulation was also performed, to validate the code. For this purpose the inflow perturbations were put to zero. Results of the progress variable of the laminar Bunsen flame are shown in Fig. 3b. This simulation was performed with the same grid size $h=0.1 \mathrm{~mm}$ as the turbulent case. The number of grid cells equalled $4 \times 256 \times 512$ (there were no variations in the $x$-direction as the flame is two-dimensional). As a validation we computed the burning velocity of the laminar flame,

$$
s=\frac{\int \rho u_{z}(1-c) d y}{\rho_{0} L_{c}}=\frac{0.0256}{1.15 \cdot 0.0626}=0.362 \mathrm{~m} / \mathrm{s},
$$

The integral was taken across the burner exit at $z=0$ (limits in the $y$-direction were determined by $\left.c=c_{1}\right), L_{c}$ is the length of the contour $c=c_{1}$ inside the flow domain. For the laminar flame the contours are close to each other (Fig. 3b); the values in the equation above correspond to level $c_{1}=0.6$. The value of $s$ is only $1 \%$ lower than the flamelet value $s_{L 0}=0.366 \mathrm{~m} / \mathrm{s}$, thus the validation was successful.

The turbulence generated by these inflow conditions quickly developed, and at $z=1 \mathrm{~mm}$ it was characterized by a turbulent intensity of $0.6 \mathrm{~m} / \mathrm{s}$, a Taylor lengthscale $\lambda$ of $1.4 \mathrm{~mm}$, such that $R e_{\lambda}=50$. The Kolmogorov length-scale at this location equalled $0.09 \mathrm{~mm}$, which was properly resolved by the DNS grid. Centerline mean velocity, turbulence intensities, and turbulence length-scales are shown in Fig. 4. The turbulent flame was verified to be in the thin reaction zone regime, with the inflow $u^{\prime} / s_{L 0}$ about 1.6 and the Karlovitz number around 16 (ratio of the gradient thickness of the progress variable and the Kolmogorov length, $u^{\prime}$ is the turbulent intensity, $s_{L 0}=0.366 \mathrm{~m} / \mathrm{s}$ is the laminar burning velocity). The turbulent burning velocity was about $2.5 s_{L 0}$.
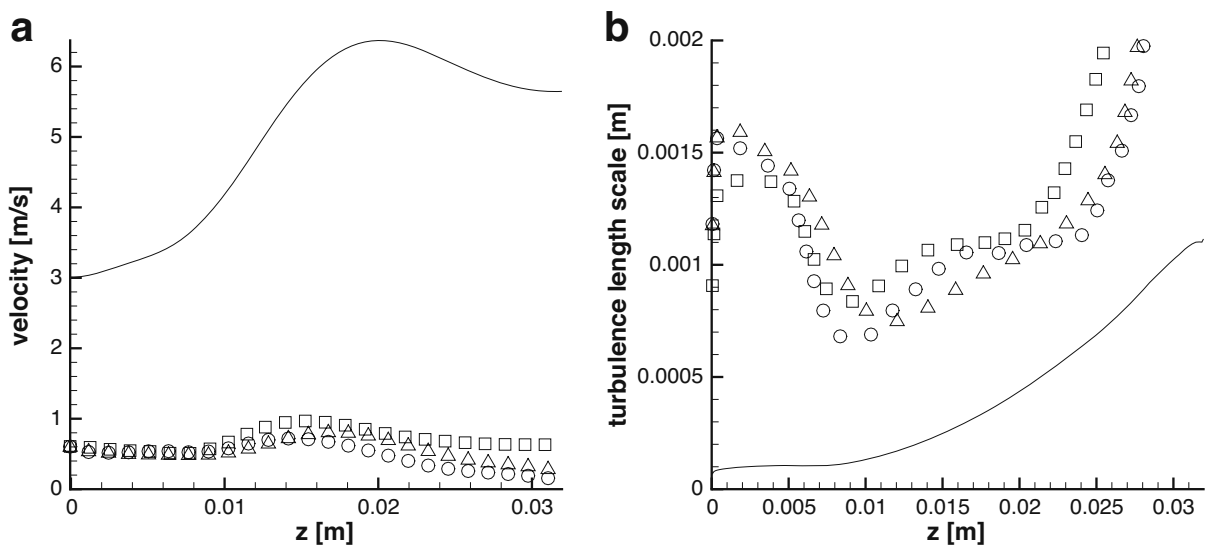

Fig. 4 a Centerline mean streamwise velocity (solid) and streamwise (square), normal (circle) and spanwise (triangle) turbulence intensities. b Centerline Kolmogorov length-scale (solid) and Taylor micro-scales based on streamwise (square), normal (circle) and spanwise (triangle) velocity component 
Table 1 Overview of simulations of turbulent Bunsen flame

\begin{tabular}{|c|c|c|c|c|c|}
\hline Simulation & $\begin{array}{l}\Delta \\
{[\mathrm{mm}]}\end{array}$ & $\begin{array}{l}h \\
{[\mathrm{~mm}]}\end{array}$ & $\begin{array}{l}\text { Source-term } \\
\text { subgrid model }\end{array}$ & $\begin{array}{l}\delta t \\
{\left[10^{-5} \mathrm{~s}\right]}\end{array}$ & $\begin{array}{l}\text { Line in } \\
\text { Figs. } 6,7\end{array}$ \\
\hline DNS & - & 0.1 & - & 0.1 & Thick solid \\
\hline LESO & 0.8 & 0.8 & - & 2.0 & Long dash \\
\hline LES1a & 0.8 & 0.8 & Similarity & 2.0 & Thin solid \\
\hline LES1b & 0.8 & 0.4 & Similarity & 1.0 & Dashed \\
\hline LES1c & 0.8 & 0.2 & Similarity & 0.5 & Dash-dotted \\
\hline LES2 & 0.4 & 0.4 & Similarity & 1.0 & Dotted \\
\hline LES3 & 0.8 & 0.8 & $\beta$-pdf & 2.0 & Thin solid \\
\hline
\end{tabular}

Six LES were performed, with uniform cubical grid cells. An overview of these simulations is provided in Table 1. The coarsest LES was at least three orders of magnitude less expensive than the DNS, because in each spatial direction the mesh was 8 times coarser than in the DNS and the time-step was 10 times larger.

\subsection{Comparison between LES and DNS}

The LES results are discussed in the following five paragraphs. First, we discuss the LES results along the first line of grid refinement ( $h$ decreases with $\Delta=h$ ). Second, we discuss the LES-results along the second line of grid refinement $(h$ decreases and $\Delta$ remains constant). Third, we demonstrate the effect of the similarity reaction subgrid model, by comparison with a simulation in which reaction subgrid modeling was ignored. Fourth, we compare the similarity reaction subgrid model with the presumed $\beta$-pdf model. Fifth, we explain how the results prompted us to formulate the similarity/deconvolution model at $2 \Delta$ instead of $\Delta$.

First, LES results along the $\Delta=h$ refinement strategy are shown in Figs. 5 and 6 a along DNS results. The snapshots (Fig. 5) show that, compared to DNS, both the

a

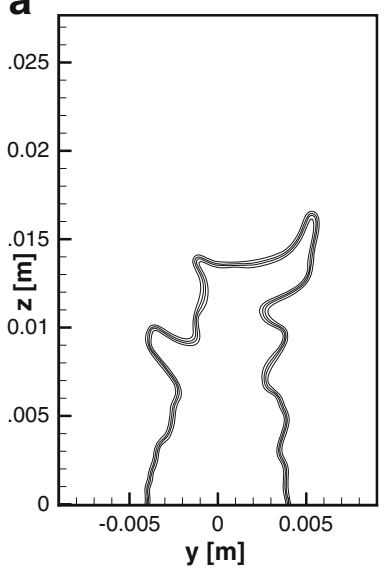

b

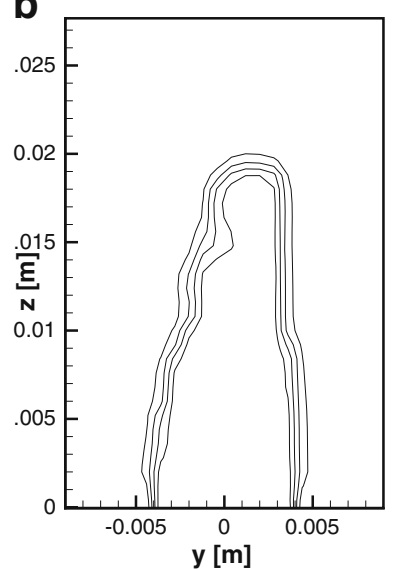

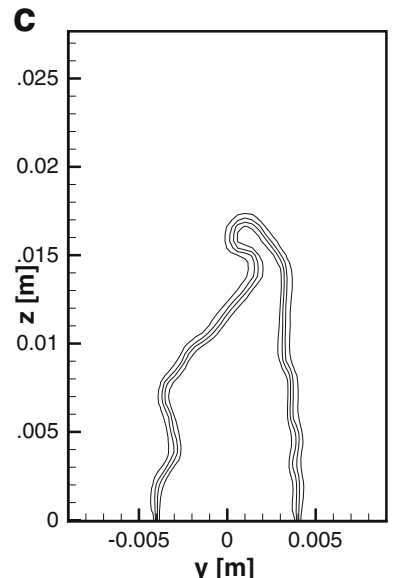

Fig. 5 Snapshots of progress variable in the plane $x=0$ at $t=0.2 \mathrm{~s}$. Isocontours $0.2,0.4,0.6$ and 0.8 . DNS (a); LES1a $h=\Delta=0.8 \mathrm{~mm}(\mathbf{b}) ; \operatorname{LES} 2 h=\Delta=0.4 \mathrm{~mm}$ (c) 
a

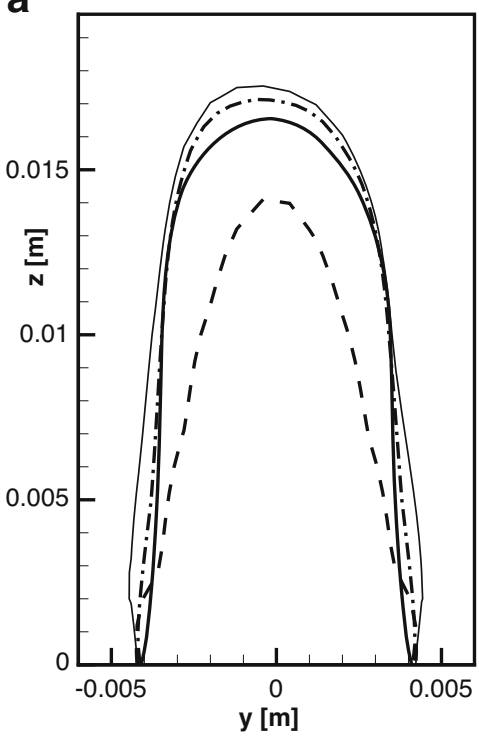

b

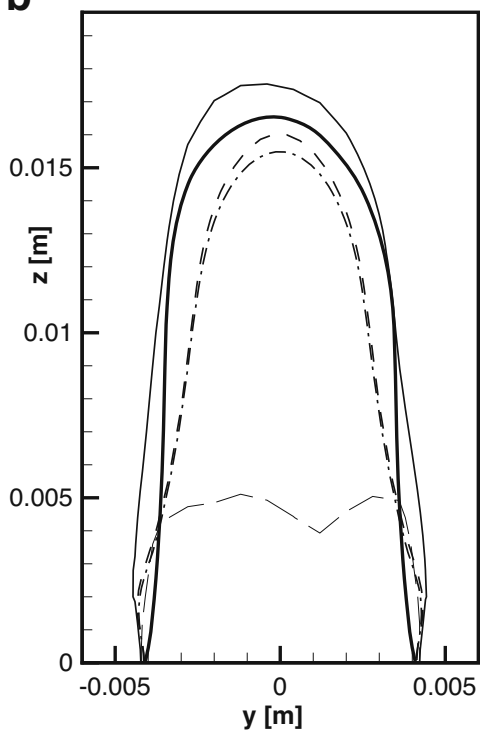

Fig. 6 Isocontour 0.75 of the mean progress variable: a refinement strategy $\Delta=h$ for similarity model and one result for $\beta$-pdf model; b refinement strategy for similarity model with $\Delta$ held fixed, the effect of no subgrid source term is also included here. DNS (thick solid); LES1a (thin solid, $h=\Delta=0.8 \mathrm{~mm}$ ); LES2 (thick dash-dotted, $h=\Delta=0.4 \mathrm{~mm}$ ); LES3 (thick dashed, $h=\Delta=0.8 \mathrm{~mm}$, $\beta$-pdf model); LES1b (dashed, $h=\Delta / 2=0.4 \mathrm{~mm}$ ); LES1c (dash-dotted, $h=\Delta / 4=0.2 \mathrm{~mm}$ ); LES0 (long dash, $h=\Delta=0.8 \mathrm{~mm}$, no subgrid source term)

gradient of the scalar and the amount of wrinkling are reduced in the LES. Both reduce with increasing $\Delta$. The mean statistics of the LES with the finer resolution (LES2) are most accurate according to Fig. 6a. In this figure time-averaged scalar contours for $c=0.75$, the contour of maximum source term (see Fig. 1c).

Second, we not only varied the mesh-size $h$ with $\Delta / h$ held fixed (equal to one), but we also investigated the accuracy of the LES by refining the grid with $\Delta$ kept constant (LES1a-c), see Fig. 4b. The results of LES1b and LES1c are almost the same, which indicates that the discretization errors are not large for these cases with $h \leq \Delta / 2$. Thus the difference between LES1bc and DNS is an estimate for the modeling errors. However, discretization errors are not small in LES1a, as there is significant deviation between LES1a and the more resolved simulations LES1bc. It appears that LES1a predicts the mean source term better than LES1bc do (Fig. 5b). This illustrates that for this quantity the significant discretization error on the coarse grid cancels the modeling error to some extent [7,8]. Such a behavior is not necessarily caused by the closure of the chemical source term, since for unreactive turbulent flows it was shown before that the LES-predictions for $h=\Delta$ can be better than for $h=\Delta / 2$ ( $\Delta$ fixed) [7]. This was found for second-order spatial accuracy only; for fourth-order methods $h=\Delta$ results were less accurate than those obtained for $h=\Delta / 2$ ( $\Delta$ fixed) [7].

Third, Figs. $4 \mathrm{~b}$ and 7 show a very large effect of the subgrid modeling of the source term. LES performed very poorly when no subgrid model was employed for the source term (LES0, where $\omega(\tilde{c})$ was used to model $\overline{\omega(c)})$; The long-dash line in Fig. 7b shows that LES0 predicted a flame which was much too short and hardly came off the 

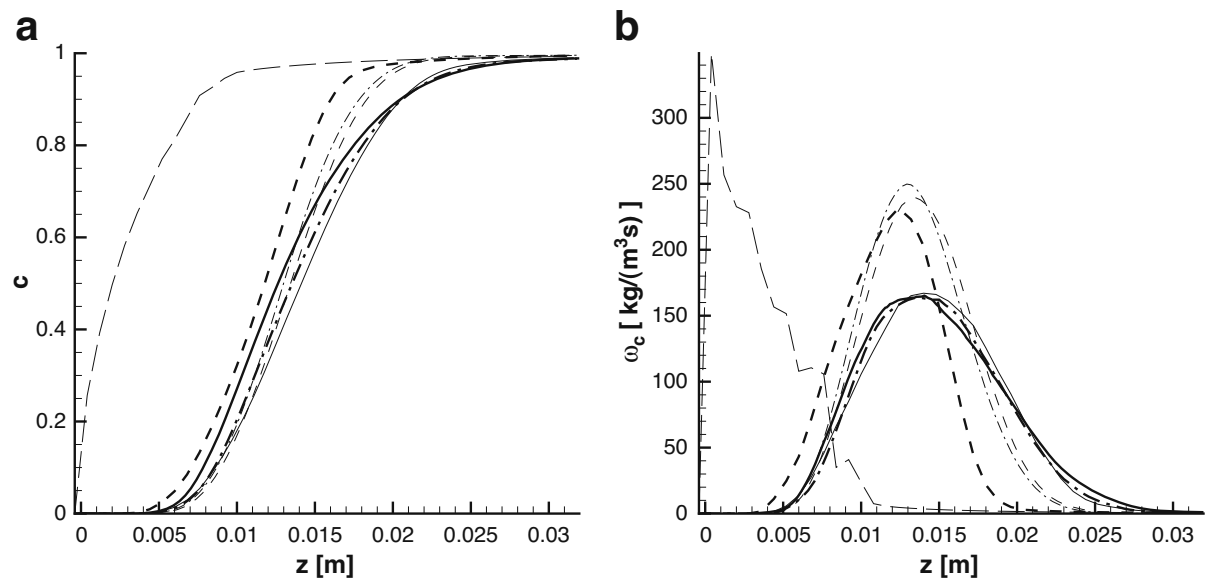

Fig. 7 Centerline statistics; mean progress variable (a) and mean source term (b) (subgrid modeling included where applicable). DNS (thick solid); LES0 (long dash, $h=\Delta=0.4 \mathrm{~mm}$, no subgrid source term), LES1a (thin solid, $h=\Delta=0.8 \mathrm{~mm}$ ), LES1b (dashed, $h=\Delta / 2=0.4 \mathrm{~mm}$ ), LES1c (dash-dotted, $h=\Delta / 4=0.2 \mathrm{~mm})$, LES2 (thick dash-dotted, $h=\Delta=0.4 \mathrm{~mm})$, LES3 (thick dashed, $h=\Delta=0.8 \mathrm{~mm}, \beta$-pdf model)

burner, represented by the inflow condition. The subgrid eddy-viscosity and subgrid eddy-diffusivity were still switched on in this case.

The fourth issue in this results section addresses the comparison between the present similarity subgrid model and the standard presumed $\beta$-pdf approach. The latter model produces a flame that is somewhat too short (Fig. 6a) and a source term that is too high (Fig. 7b). On the whole, comparing similarity simulation LES1a and $\beta$-pdf simulation LES3, the former produces slightly better results. For another value of the model coefficient in the $\beta$-pdf model, $a=0.33$ instead of $a=0.14$, results became worse (not shown). In addition we compare the similarity and $\beta$-pdf model with respect to the fractions of grid points for which manifold extrapolation was required. The quantity $2 \tilde{c}-J \tilde{c}$ was larger (smaller) than $1.01(-0.01)$ in $1.5 \%$ $(0.3 \%)$ of the grid points in case of LES1a, 3.0\% $(1.4 \%)$ in case of LES1b, $3.9 \%$ $(1.7 \%)$ in case of LES1c, and $0.14 \%(0.09 \%)$ in case of the finer grid case LES 2 . The subgrid variance model in $\beta$-pdf case LES3 with $a=0.14$ attained values larger than $1.01 \tilde{c}(1-\tilde{c})$ in $11 \%$ of the grid points. If we compare the fractions of LES1a with those of LES3, we conclude that again the similarity model performs somewhat better than the $\beta$-pdf model, since the similarity model enters the extrapolated region of the manifold less frequently.

Finally, it is remarked that, in order to obtain acceptable LES results on the coarse grid, it was necessary to apply the source-term similarity model at the scale $2 \Delta$ (and not at $\Delta$ ). We shortly describe what happened when we applied the similarity model at the scale $\Delta$ (for the case with $\Delta=h=0.8 \mathrm{~mm}$ ). Since then the filterwidth of $H$ equalled the grid size, it was convenient to approximate $H$ with a truncated Taylor expansion, $H \approx I+\left(\Delta^{2} / 24\right) \nabla^{2}$. However, the flame resulting from that simulation turned out to be even shorter than the result of LES0, in which no model for the subgrid source term was used. Thus like LES0, this simulation erroneously predicts a high risk of flash-back in an experimental flame with similar flow parameters. As $H$ with filterwidth $\Delta$ appeared to be inappropriate for the present case, we started to 
use $H$ with filterwidth $2 \Delta$. This choice turned out to be quite beneficial. The formal justification to use $2 \Delta$ instead of $\Delta$ is that a deconvolution can never retrieve all scales in actual LES, simply because the finest physical scales cannot be recognized by the grid. Here we deliberately used a deconvolution step with a 'wrong' filterwidth to 'overrelax' the scales larger than $\Delta$, compensating for the missing unretrievable scales. Apparently, this was a successful strategy for the Bunsen flame application.

\section{Conclusions}

LES with premixed flamelet chemistry were compared with DNS. The test-case was a premixed Bunsen flame with turbulent inflow characterized by $R e_{\lambda}=50$. This DNS, resolved down to the Kolmogorov scale, was used to test LES at much coarser grids. A similarity (or first-step deconvolution model) was used to include subgrid reaction effects. It was found to be important to apply the similarity model at $2 \Delta$, in order to compensate for the unretrievable subgrid scales. When the model was applied at the basic filterwidth, flash-back occurred (the flame did not come off the burner, represented by the inflow condition). A similar thing occurred when subgrid reaction effects were ignored altogether. However, when they were included with the similarity model at scale $2 \Delta$, reasonable results were obtained. A drawback of the model is that physical upperbound of the scalar is violated (overshoots of about $20 \%$ ). However, sofar this drawback has no serious implications; an additional simulation in which the scalar was clipped at each time-step to satisfy the physical constraint provided almost the same results.

The accuracy of LES with the similarity model was investigated in detail. Two types of grid refinements were considered: grid refinement where both $h$ and $\Delta$ were decreased; and grid refinement where $h$ was decreased and $\Delta$ stayed constant. The first type of grid refinement altered the mean scalar statistics only slightly. The second type of grid refinement, which had larger effect, serves to distinguish between discretization and modeling errors. It appeared that discretization errors had considerable influence for $h=\Delta$, but not necessarily a bad influence, since for the chemical source term for example, the results for $h=\Delta$ were closer to the DNS than for $h=\Delta / 2$. Results for $h=\Delta / 2$ and $h=\Delta / 4$ were quite similar, which indicates that for $h \leq \Delta / 2$ the effects of discretization errors were small.

The similarity model for subgrid chemistry was also shown to perform slightly better than standard $\beta$-pdf modeling, for this flame.

Acknowledgement This research was funded by Stichting Technische Wetenschappen, grant number EWO.5874.

Open Access This article is distributed under the terms of the Creative Commons Attribution Noncommercial License which permits any noncommercial use, distribution, and reproduction in any medium, provided the original author(s) and source are credited.

\section{References}

1. Peters, N.: Turbulent Combustion. Cambridge University Press, Cambridge (2000)

2. Pitsch, H., Steiner, H.: Large-eddy simulation of a turbulent piloted methane/air diffusion flame (Sandia flame D). Phys. Fluids 12, 2541-2554 (2000) 
3. Van Oijen, J.A., De Goey, L.P.H.: Modelling of premixed laminar flames using flameletgenerated anifolds. Combust. Sci. Technol. 161, 113-137 (2000)

4. Van Oijen, J.A.: Flamelet-generated manifolds: development and application to premixed laminar flames. Ph.D. thesis, University of Technology Eindhoven (2002)

5. Pierce, C.D., Moin, P.: Progress-variable approach for large-eddy simulation of non-premixed turbulent combustion. J. Fluid Mech. 504, 73-97 (2004)

6. Domingo, P., Vervisch, L., Payet, S., Hauguel, R.: DNS of a premixed turbulent V flame and LES of a ducted flame using a FSD-PDF subgrid scale closure with FPI-tabulated chemistry. Combust. Flame 143, 566-586 (2005)

7. Vreman, B., Geurts, B., Kuerten, H.: Comparison of numerical schemes in large-eddy simulation of the temporal mixing layer. Int. J. Numer. Methods Fluids 22, 297-311 (1996)

8. Meyers, J., Geurts, B.J., Baelmans, M.: Database analysis of errors in large-eddy simulation. Phys. Fluids 15, 2740-2755 (2003)

9. Filatyev, S.A., Driscoll, J.F., Carter, C.D., Donbar, J.M.: Measured properties of turbulent premixed flames for model assessment, including burning velocities, stretch rates, and surface densities. Combust. Flame 141, 1-21 (2005)

10. Bell, J.B., Day, M.S., Grcar, J.F., Lijewski, M.J., Driscoll, J.F., Filatyev, S.A.: Numerical simulation of a laboratory-scale turbulent slot flame. Proc. Comb. Inst. 31, 1299-1307 (2007)

11. Van Oijen, J.A., Bastiaans, R.J.M., Groot, G.R.A., de Goey, L.P.H.: Direct numerical simulations of premixed turbulent flames with reduced chemistry: validation and flamelet analysis. Flow Turbul. Combust. 75, 67-84 (2005)

12. Givi, P.: Model-free simulations of turbulent reactive flows. Prog. Energy Combust. Sci. 15, 1-107 (1989)

13. Pope, S.B.: Ten questions concerning the large-eddy simulation of turbulent flows. New J. Phys. 6, 35 (2004)

14. Smooke, M.D., Giovangigli, V.: Formulation of the premixed and nonpremixed test problems. In: Smooke, M.D. (ed.) Reduced kinetic mechanisms and asymptotic approximations for methaneair flames, pp. 1-28. Springer, Berlin (1991)

15. Vreman, A.W.: An eddy-viscosity model for turbulent shear-flow: algebraic theory and applications. Phys. Fluids 16, 3670-3681 (2004)

16. Vreman, A.W., van Oijen, J.A., de Goey, L.P.H., Bastiaans, R.J.M.: Subgrid scale modeling in large eddy simulation of turbulent combustion using premixed flamelet chemistry. Flow Turbul. Combust. (2008). doi:10.1007/s10494-008-9159-x

17. Geurts, B.J.: Regularization modeling for large-eddy simulation of diffusion flames. In: Proceedings ECCOMAS CFD 2006, Delft University of Technology, Delft, 5-8 September 2006

18. Bardina, J., Ferziger, J.H., Reynolds, W.C.: Improved turbulence models based on LES of homogeneous incompressible turbulent flows. Department of Mechanical Engineering, Report No TF-19, Stanford (1984)

19. Stolz, S., Adams, N.A., Kleiser, L.: An approximate deconvolution model for large-eddy simulation with application to incompressible wall-bounded flows. Phys. Fluids 13, 997-1015 (2001)

20. Vreman, B., Geurts, B., Kuerten, H.: Realizability conditions for the turbulent stress tensor in large-eddy simulation. J. Fluid. Mech. 278, 351-364 (1994)

21. Cook, A.W., Riley, J.J.: A subgrid model for equilibrium chemistry in turbulent flows. Phys. Fluids 6, 2868-2870 (1994)

22. Wall, C., Boersma, B.J., Moin, P.: An evaluation of the assumed beta probability density function subgrid-scale model for large eddy simulation of nonpremixed turbulent combustion with heat release. Phys. Fluids 12, 2522-2529 (2000)

23. Vreman, A.W., Albrecht, B.A., van Oijen, J.A., de Goey, L.P.H., Bastiaans, R.J.M.: Premixed and non-premixed generated manifolds in large-eddy simulation of Sandia flame D and F. Combust. Flame 153, 394-416 (2008)

24. van der Burg, J.W.: Numerical techniques for transonic flow calculations. Ph.D. thesis, University of Twente (1993)

25. Wesseling, P.: An Introduction to Multigrid Methods. Wiley, New York (1992) 